\title{
A diverse global fungal library for drug discovery
}

\author{
Guodong Niu ${ }^{1}$, Thirunavukkarasu Annamalai ${ }^{2}$, Xiaohong Wang ${ }^{1}$, Sheng Li ${ }^{3}$, Stephen Munga ${ }^{4}$, Guoming Niu ${ }^{5}$, \\ Yuk-Ching Tse-Dinh ${ }^{2,6}$, Jun Li ${ }^{\text {Corresp. 1, } 6}$ \\ 1 Department of Biological Sciences, Florida International University, Miami, FL, United States \\ 2 Department of Chemistry and Biochemistry, Florida International University, Miami, FL, United States \\ 3 College of Public Health, City University of New York (CUNY)System, New York, United States \\ ${ }^{4}$ Center for Global Health Research, Kenya Medical Research Institute, Kisumu, Kenya \\ 5 Department of Hematology, Southern Medical University Affiliated Nanhai Hospital, Foshan, Guangdong, China \\ 6 Biomolecular Sciences Institute, Florida International University, Miami, FL, United States \\ Corresponding Author: Jun Li \\ Email address: lij@fiu.edu
}

Background. Secondary fungal metabolites are important sources for new drugs against infectious diseases and cancers. Methods. To obtain a library with enough diversity, we collected about 2395 soil samples and 2324 plant samples from 36 regions in Africa, Asia, and North America. The collection areas covered various climate zones in the world. We examined the usability of the global fungal extract library (GFEL) against parasitic malaria transmission, Gram-positive and negative bacterial pathogens, and leukemia cells.

Results. Nearly ten thousand fungal strains were isolated. Sequences of nuclear ribosomal internal transcribed spacer (ITS) from 40 randomly selected strains showed that over $80 \%$ were unique. Screening GFEL, we found that the fungal extract from Penicillium thomii was able to block $P$. falciparum transmission to Anopheles gambiae, and the fungal extract from Tolypocladium album was able to kill myelogenous leukemia cell line K562. We also identified a set of candidate fungal extracts against bacterial pathogens. 
2 Guodong Niư ${ }^{1}$, Thirunavukkarasu Annamalai ${ }^{2}$, Xiaohong Wang ${ }^{1}$, Sheng $\mathrm{Li}^{3}$, Stephen Munga ${ }^{4}$,

3 Guomin Niư ${ }^{5}$, Yuk-Ching Tse-Dinh ${ }^{2,6}$, and Jun $\mathrm{Li}^{1,6^{*}}$

4

$5{ }^{1}$ Department of Biological Sciences, ${ }^{2}$ Department of Chemistry and Biochemistry, ${ }^{6}$

6 Biomolecular Sciences Institute, Florida International University, Miami, Florida, USA

$7 \quad{ }^{3}$ College of Public Health, New York City University, New York, New York, USA

$8{ }^{4}$ Center for Global Health Research, Kenya Medical Research Institute, Kisumu, Kenya

$9{ }^{5}$ Department of Hematology, Southern Medical University Affiliated Nanhai Hospital,

10 Guangzhou, Guangdong, China

11

12

$13{ }^{*}$ Corresponding Author:

14 Jun Li

1511200 SW 8th St, OE 214

16 Email address: lij@fiu.edu

17

18 


\section{Abstract}

20 Background. Secondary fungal metabolites are important sources for new drugs against

21 infectious diseases and cancers.

22 Methods. To obtain a library with enough diversity, we collected about 2395 soil samples and 232324 plant samples from 36 regions in Africa, Asia, and North America. The collection areas 24 covered various climate zones in the world. We examined the usability of the global fungal 25 extract library (GFEL) against parasitic malaria transmission, Gram-positive and negative 26 bacterial pathogens, and leukemia cells.

27 Results. Nearly ten thousand fungal strains were isolated. Sequences of nuclear ribosomal 28 internal transcribed spacer (ITS) from 40 randomly selected strains showed that over $80 \%$ were 29 unique. Screening GFEL, we found that the fungal extract from Penicillium thomii was able to 30 block $P$. falciparum transmission to Anopheles gambiae, and the fungal extract from 31 Tolypocladium album was able to kill myelogenous leukemia cell line K562. We also identified a 32 set of candidate fungal extracts against bacterial pathogens. 
34

35

36

37

38

\section{Introduction}

Natural products, produced by living organisms in nature, have been used as medicine for thousands of years (Dias, Urban et al. 2012, Buyel 2018). For instance, the treatment of malaria was recorded in China with the Qinghao plant (Artemisia annua) as early as the second century. The active ingredient, qinghaosu (artemisinin), was isolated from the plant by Youyou Tu and her colleagues in 1971 (Luo and Shen 1987, Hsu 2006). The establishment of microbiology in the early modern era led to drug discoveries from microbes. The first antibiotics, penicillin, was discovered from a fungus by Alexander Fleming (Fleming 1929). Fungi have initially been and still are used to produce medicines to treat infectious diseases (Elder 1944). Furthermore, people use natural products to treat tumors. Indeed, more than half of anti-tumor drugs or leads in current clinical trials are from natural products (Wolfender and Queiroz 2012). Microbial metabolites are essential resources for drug discovery (Lenzi, Costa et al. 2018). Compared with other natural product resources, fungi have the following advantages. First, there are enormous fungal species: about 120,000 fungal species have been described (Hawksworth and Lucking 2017) and 5.1 million fungal species are estimated (Blackwell 2011). Second, fungi produce broad and diverse secondary metabolites with a vast difference in chemical structures (Pham, Yilma et al. 2019). Third, large-scale fermentation can generate a large amount of fungal secondary metabolites, which was exampled by the production of alcohol and lactic acid. However, yield of many target fungal secondary metabolites is restricted by fungal growth and differentiation (Nielsen and Nielsen 2017, Keller 2019, Pham, Yilma et al. 2019), which is resolved by new technologies that enable us to engineer a fungus to produce a specific compound in high yield by modifying its metabolic pathways (van Dijk and Wang 2016).

Also, the recent development of genomic sequencing technology and the identification of more biosynthetic gene clusters accelerate the discovery and application of new compounds from fungi (Hussain, Al-Sadi et al. 2017, Keller 2019). 
Fungi can be isolated from soil, water, air, plants, or other organisms. In particular, the

61 endophytic fungi from the plants can generate similar secondary metabolites as their hosts

62 (Venieraki, Dimou et al. 2017). Thus, the heterologous expression can replace their hosts as the

63 supplies of crude materials for some medicines (van Dijk and Wang 2016). Diverse fungal

64 libraries are critical for the research and industry communities(Niu, Wang et al. 2015). At

65 present, there are several specific fungal libraries for drug discovery (May, Milne et al. 2004,

66 Richards, Jones et al. 2012, Zhang, Tang et al. 2014), many of which focus on specific

67 environments (Li, Qing et al. 2005, Gonzalez-Menendez, Crespo et al. 2018, Zhang, Li et al.

68 2018). We focus on generating a global diverse fungal library to facilitate new drug discovery.

69 The soil and plant samples were collected globally, currently including from Asia, Africa, and

70 North America.

71 To determine the usability of our fungal library, we screened the newly established

72 fungal extract library for malaria transmission inhibitors, antibiotics, and drug leads against

73 chronic myeloid leukemia. A set of positives were discovered.

74

75 Materials \& Methods

76 Collecting samples: We collected plant and soil samples from different regions around the 77 world. The field collection was approved by the United States Department of Agriculture with 78 permit number of P526P-18-03319. The soil samples were taken in 5-10 cm depths under the 79 surface. Plant samples consisted of the whole plant or separated plant parts such as roots, 80 stems, leaves, flowers, fruits, or various combinations of components. Samples were stored on 81 ice or in a $4^{\circ} \mathrm{C}$ fridge immediately after collection. No samples were more than $5 \mathrm{~g}$. Most of 82 samples collected by authors, residents, and friends were mailed to labs and processed locally.

83 For instance, samples collected in China and Myanmar were shipped to Guomin Niu's lab in 84 Guangdong, China to process. Samples collected in the US were processed Jun Li's lab in 85 Florida, USA. The fungal extracts form the GFEL library. 
86 Isolation of fungi from soil and plants: For each soil sample, we transferred $50 \mathrm{mg}$ soil to a

$871.5 \mathrm{~mL}$ plastic tube, and $1 \mathrm{~mL}$ autoclaved distilled water was added. The sample was vortexed 88 for 30 secs and centrifuged at $500 \mathrm{~g}$ for 2 minutes $(\mathrm{min})$ to get rid of the soil particles. For plant 89 samples, the first step was to sterilize the plant surface by rinsing the sample with distilled $\mathrm{H}_{2} \mathrm{O}$, 90 then soaking in $70 \%$ ethanol for 10 seconds (sec) and rinsing again with distilled $\mathrm{H}_{2} \mathrm{O}$. After 91 sterilization, the plants were cut into $0.5 \mathrm{~cm} \times 0.5 \mathrm{~cm}$ pieces and transferred into a sterile mortar.

92 Then, $2 \mathrm{~mL}$ of distilled $\mathrm{H}_{2} \mathrm{O}$ was added, followed by grinding with a pestle for 1-3 min and the 93 slurry was transferred to a $1.5 \mathrm{~mL}$ plastic tube and then centrifuged at $500 \mathrm{~g}$ for $2 \mathrm{~min}$ to get rid 94 of the particles. About $100 \mu \mathrm{L}$ of upper supernatant from treated soil or plants was evenly 95 spread onto a $100 \times 15 \mathrm{~mm}$ Petri Dish plate containing $14 \mathrm{~mL}$ Malt Extract Agar (MEA), made of $9610 \mathrm{~g}$ malt extract, $1 \mathrm{~g}$ yeast extract, $15 \mathrm{~g}$ agar, and $0.05 \mathrm{~g}$ chloramphenicol (Sigma-Aldrich, St. 97 Louis, $\mathrm{MO}$ ) in $1 \mathrm{~L}$ distilled $\mathrm{H}_{2} \mathrm{O}$ and autoclaved at $121^{\circ} \mathrm{C}$ for $20 \mathrm{~min}$. The plates were sealed with 98 parafilm, and the fungi were allowed to grow for 7-14 days at room temperature (RT) with cycles of 12 hours (hr) of darkness and $12 \mathrm{hr}$ of light.

The fungal colonies on the MEA medium plates were picked with a toothpick and inoculated in a new MEA plate by streaking. If the colonies were mixtures of two or more species, we kept inoculating and streaking until a single colony appeared. Finally, a piece of fungal agar containing mycelium or spores was cut and transferred to a $1.5 \mathrm{~mL}$ Eppendorf tube containing $500 \mu \mathrm{L}$ of sterile $20 \%$ glycerol in distilled $\mathrm{H}_{2} \mathrm{O}$. We stored the cells in a $-80{ }^{\circ} \mathrm{C}$ freezer for long-term storage.

Metabolite production and extraction: Cereal based medium was used to grow fungi to 107 produce secondary metabolites (Niu, Wang et al. 2015). Briefly, six pieces of Cheerios 108 Breakfast cereals (General Mills, Minneapolis, MN) were placed to a glass test tube, capped 109 with a plastic lid and autoclaved for $20 \mathrm{~min}$, and then $2 \mathrm{~mL}$ sterile sucrose water $(3 \mathrm{~g}$ of sucrose 110 and $50 \mathrm{mg}$ chloramphenicol in $1 \mathrm{~L}$ distilled $\mathrm{H}_{2} \mathrm{O}$ ) was added into the tube. Later, the fungal 111 colony grown on the MEA plate was inoculated into the cereal medium and incubated at RT for 
112 one month to produce sufficient metabolites. A month late, $2 \mathrm{~mL}$ ethyl acetate was added into a

113 tube to extract the fungal metabolites, mixed with a glass stirring rod, and placed overnight in a

114 chemical hood with gentle shaking. The next day, $1 \mathrm{~mL}$ upper layer of supernatant was

115 transferred to a $1.5 \mathrm{~mL}$ tube. After centrifugation (2, $000 \mathrm{~g}$ for $2 \mathrm{~min})$, around $950 \mu \mathrm{L}$ clear

116 supernatant was transferred to a pre-weighed $1.5 \mathrm{~mL}$ plastic tube and dried with SpeedVac

117 concentrator (Thermo Fisher Scientific, Waltham, MA). Finally, the dry extracts were weighed

118 and dissolved in an appropriate amount of dimethyl sulfoxide (DMSO) to prepare $10 \mathrm{mg} / \mathrm{mL}$

119 stock solution and stored in a $-20^{\circ} \mathrm{C}$ freezer for future screening assays.

120 Determination of fungal species: Forty fungal isolates were randomly picked to evaluate the

121 fungal library's diversity. The fungi were cultured with liquid malt extract medium at RT for one week, and mycelium was collected for DNA extraction using DNAzol (Thermo Fisher). Genomic DNA applied as PCR templates were isolated using DNAzol Reagent following the manual

124 (Thermo Fisher Scientific). To identify the fungal species, nuclear ribosomal ITS regions were amplified with by PCR with specific primers (Table 1) (Op De Beeck, Lievens et al. 2014) using the following approach: $94^{\circ} \mathrm{C} 2 \mathrm{~min} ; 94^{\circ} \mathrm{C} 30 \mathrm{sec}, 55^{\circ} \mathrm{C} 30 \mathrm{sec}, 72^{\circ} \mathrm{C} 1 \mathrm{~min}, 35$ cycles; $72{ }^{\circ} \mathrm{C} 5$ min. The amplified products were sequenced and blasted against the NCBI database to identify fungal species (Raja, Miller et al. 2017). with ELISA assays: As described previously (Niu, Wang et al. 2015), the red blood cells (iRBC) infected by Plasmodium falciparum (NF54 strain from MR4, Manassas, VA) were cultured in RPMI-1640 medium (Life Tech, Grand Island, NY) supplemented with 10\% heatinactivated (56 ${ }^{\circ} \mathrm{C}$ for $45 \mathrm{~min}$ ) human $\mathrm{AB}+$ serum (Interstate blood bank, Memphis, TN), 12.5 $\mu \mathrm{g} / \mathrm{mL}$ hypoxanthine and $4 \%$ hematocrit $\left(\mathrm{O}+\right.$ human blood) in a candle jar at $37^{\circ} \mathrm{C}$ for $15-17$ days. The medium was replaced every day to provide sufficient nutrients. Blood smears stained with Giemsa (Sigma-Aldrich, St. Louis, MO) were used to examine parasitemia or

137 gametocytemia every other day under a light microscope. Then, the cells were collected and 
138

139

140

141

142

143

144

145

146

147

148

149

150

151

152

153

154

155

156

157

158

159

160

161

162

washed three times with RPMI-1640 at $300 \times \mathrm{g}$ for $4 \mathrm{~m}$. The cell pellets were re-suspended in PBST (PBS containing $0.2 \%$ Tween-20) and homogenized by ultra-sonication with six cycles of $10 \mathrm{sec}$ pulse and $50 \mathrm{sec}$ resting on ice for each period. The lysates were centrifuged at $8,000 \mathrm{~g}$ for $2 \mathrm{~min}$ to remove insoluble materials and cellular debris. With the iRBC lysate and insect cellexpressed recombinant FREP1, the ELISA assay was used to screen the fungal extract library to block FREP1-parasite interaction (Niu, Wang et al. 2015). A 96-well ELISA plate was coated with $50 \mu \mathrm{L}$ iRBC lysate $\left(2 \mathrm{mg} / \mathrm{mL}\right.$ protein) overnight at $4^{\circ} \mathrm{C}$. After coating, the plate was blocked with $100 \mu \mathrm{L}$ of PBS plus $0.2 \%$ bovine serum albumin (BSA) per well for $1.5 \mathrm{hr}$ at RT. After removal of the blocking solution, FREP1 $(10 \mu \mathrm{g} / \mathrm{mL})$ in blocking buffer (PBS plus $0.2 \%$ BSA) was added to each well, and $1 \mu \mathrm{L}$ fungal extract was taken from a 96-well plate containing 2 $\mathrm{mg} / \mathrm{mL}$ crude extract dissolved in DMSO in each well with a multiple-channel pipette and transferred to the ELISA plate, then incubated for $1 \mathrm{hr}$ at RT with gentle shaking. After washing three times with PBST, $50 \mu \mathrm{L}$ rabbit anti-FREP1 polyclonal antibody (Niu et al., 2015) (diluted 1: 5,000 in blocking buffer, $1 \mu \mathrm{g} / \mathrm{mL}$ ) was added to each well and incubated for $1 \mathrm{hr}$ at RT. About $50 \mu \mathrm{L}$ alkaline phosphatase-conjugated anti-rabbit IgG (diluted 1: 20,000 in blocking buffer) was added to each well and incubated for $45 \mathrm{~min}$ at RT. The wells were washed three times with PBST between incubations. After washing, each well was developed with $50 \mu \mathrm{L}$ pNPP substrate (Sigma-Aldrich) until the colors were visible, and absorbance at $405 \mathrm{~nm}$ was measured. The functional FREP1 supplemented with $1 \mu \mathrm{L}$ solvent (DMSO) was used as non-inhibition control, and the heat-inactivated FREP1 $\left(65^{\circ} \mathrm{C}\right.$ for $15 \mathrm{~min}$ ) was used as a $100 \%$ inhibition control.

\section{Determination of the transmission-blocking activity of the fungal extracts in mosquitoes:}

Following the previous protocol (Zhang, Niu et al. 2015), the 15- to 17-day old cultured $P$. falciparum containing 2-3\% stage $\mathrm{V}$ gametocytes were collected and diluted with new $\mathrm{O}+$ type human blood to get $0.2 \%$ stage $V$ gametocytes in the blood. Then, the $150 \mu \mathrm{L}$ blood was mixed with the same volume of heat-inactivated $A B+$ human serum. Then, $3 \mu \mathrm{L}$ candidate fungal 
163 extract in DMSO (10 mg/mL or $2 \mathrm{mg} / \mathrm{mL}$ ) was mixed with $297 \mu \mathrm{L}$ infected blood, the final fungal

164 extract concentration in blood was 100 or $20 \mu \mathrm{g} / \mathrm{mL}$, respectively. SMFA was performed to feed

165 about 100 3-5 days old $A$. gambiae G3 female mosquitoes for $15 \mathrm{~min}$, and the engorged

166 mosquitoes were maintained with $8 \%$ sugar in a BSL-2 insectary $\left(28{ }^{\circ} \mathrm{C}, 12-\mathrm{h}\right.$ light/dark cycle,

$16780 \%$ humidity). The midguts were dissected seven days post-infection and stained with $0.1 \%$

168 mercury dibromofluorescein disodium salt in PBS for $16 \mathrm{~m}$. The oocysts in midguts were

169 counted under a light microscope. This standard membrane feeding assays were conducted at

170 least twice to confirm the results.

171 Screening the fungal extract library to identify antibiotics: A subset of fungal extracts,

172 randomly selected, were tested for the antibacterial activity to inhibit the growth of Shigella

173 flexneri (ATCC 9199), Staphylococcus aureus (ATCC 14775), methicillin-resistant

174 Staphylococcus aureus (MRSA, ATCC BAA-44) and E.coli (AS17tolc). The drug-screening was

175 performed in a 384-well microplate format using the following procedures. Bacteria were

176 cultured in $50 \mathrm{~mL}$ Mueller Hinton broth $(\mathrm{MHB})$ in a $150-\mathrm{mL}$ flask overnight at $37^{\circ} \mathrm{C}$. The next

177 day, the cells were first $\mathrm{OD}_{600}$ adjusted to 0.1 and then further diluted $1: 100$ in $\mathrm{MHB}$, and a

178 volume of $50 \mu \mathrm{L}\left(\sim 10^{5} \mathrm{CFU}\right)$ is added to each well of the 384-well sterile microplates (Thermo

179 Fisher Scientific). Fungal extracts $(0.5 \mu \mathrm{L}$ in DMSO) were then added to each test well at a final

180 concentration of $40 \mu \mathrm{g} / \mathrm{mL}$. The plates were incubated for $18-20 \mathrm{hr}$ at $37^{\circ} \mathrm{C}$. At the end of this

181 incubation, resazurin (Sigma-Aldrich) was added to the wells to determine the growth of

182 bacteria. The final concentration was $0.02 \%$, and the plates were further incubated for $4-6 \mathrm{hr}$ at

$18337^{\circ} \mathrm{C}$. In the presence of viable cells, resazurin was reduced to resorufin (pink) along with an

184 increase in fluorescence (O'Brien, Wilson et al. 2000). Extracts showing antibiotic activity (hits)

185 were scored as those that prevented the color change and also reduced the fluorescence (Ex

186540 , Em 590nm) by $90 \%$ when compared to the control wells containing no inhibitor.

187 Ciprofloxacin was used as a positive control for bacterial growth inhibition. Three wells were 
188 189

190

191

192

193

194

195

196

197

198

199

200

201

202

203

204

205

206

207

208

209

210

211

212

213

used for each sample. For the positive candidate extracts, we repeated the experiments at least once to confirm the results.

\section{Screening the fungal extract library to identify drugs leads against chronic myeloid}

leukemia with MTT assays: Cell proliferation was analyzed by the 3-(4,5-dimethylthiazol-2-yl)2,5-diphenyl tetrazolium bromide (MTT) using Vybrant® MTT Cell Proliferation Assay (Thermo Fisher Scientific) with the human immortalized myelogenous leukemia cell line K562. Around $2 \times 10^{4}$ cell in $100 \mu \mathrm{L}$ culture medium (RPMI $1640+2 \mathrm{mM}$ glutamine $+10 \%$ fetal bovine serum) were seeded in 96 -well microplates and incubated at $37^{\circ} \mathrm{C}$ with $5 \% \mathrm{CO}_{2}$. The next day, one $\mu \mathrm{L}$ fungal extract in DMSO was added (final concentration of the fungal extract was $20 \mu \mathrm{g} / \mathrm{mL}$ ), and the cells were incubated at $37^{\circ} \mathrm{C}$ with $5 \% \mathrm{CO}_{2}$ for another $24 \mathrm{hr}$. Next, the microplate was centrifuged at $500 \mathrm{~g}$ for $10 \mathrm{~min}$ to pellet the cells, the medium was carefully removed as much as possible, and $100 \mu \mathrm{L}$ of fresh medium was then added. About $10 \mu \mathrm{L}$ of the $12 \mathrm{mM}$ MTT stock solution was added, mixed, and incubated for $4 \mathrm{hr}$ at $37^{\circ} \mathrm{C}$. The microplate was centrifuged again at $500 \mathrm{~g}$ for $10 \mathrm{~m}$. After removing $75 \mu \mathrm{L}$ of the medium from the wells with $25 \mu \mathrm{L}$ medium with cells left, $50 \mu \mathrm{L}$ of DMSO was added to each well, mixed, and incubated at $37^{\circ} \mathrm{C}$ for $10 \mathrm{~min}$ to dissolve formazan crystal for measurement. The same amount of DMSO without drugs was applied as a control. The optical density was measured at an absorbance wavelength of 540 $n$ m. Cell growth inhibition rate $(\%)=\left(A_{540}\right.$ of control $-A_{540}$ of treatment $/ A_{540}$ of control $) \times 100 \%$. Triplicates were conducted for each sample.

Statistical analysis: All the experiments were independently repeated at least twice and analyzed with the Wilcoxon-Mann-Whitney test using GraphPad Prism (GraphPad Software, CA, USA).

Sequence availability: All fungal ITS sequences obtained in this project have been deposited into GenBank at NCBI (https://www.ncbi.nlm.nih.gov/genbank/sequenceids/).

Construction of phylogenetic tree: Forty fungal ITS sequences in FASTA format were input into an online multiple sequence alignment tool (https://www.ebi.ac.uk/Tools/msa/clustalo/) 
214 using Clustal Omega algorithm (Higgins and Sharp 1988). The parameter "DNA" was selected

215 for input sequence and "ClustalW" was selected as output format. All other parameters were

216 kept as defaults. Multiple sequence alignment was conducted by clicking on "Submit". After

217 alignment was completed, the tab of "Phylogenetic Tree" was clicked and the checkbox "Real"

218 was selected for "Branch length" to visualize the phylogenetic tree. The phylogenetic tree was

219 saved as a pdf file through "print" under "File".

220

221 Results and Discussion

222

Extensive fungal library with nearly ten thousand isolates

223

A large and diverse fungal library is powerful in discovering new drugs. To achieve this

224

goal, we collected samples globally. Current collection includes samples from Kenya, Myanmar, 225 USA, and China. We received about 2395 soil samples and 2324 plant samples. We collected 226 the whole plant or separated plant parts such as roots, stems, leaves, flowers, fruits, or various combinations of components. The samples were from 36 regions, including Nairobi in Kenya, 10 regions in the United States, Yangon in Myanmar, and 24 districts in China (Table 2). The collection places cover various climate zones.

From these samples, 9,053 fungal isolates in total were cultured. Among them, 2,356

231

232 were from the plant samples, and 6,688 were from soil samples. About one fungal strain per plant-part sample and 2.8 fungal isolates per soil sample were obtained by average. Nearly $69.4 \%$ of fungal isolates were from the subtropical climate in China (Shanghai, Guangzhou, and Chongqing) and the USA (e.g., Dallas, New Orleans, and Oklahoma City). About $8 \%$ of fungal isolates were from tropical climates such as Miami in the USA, Yangon in Myanmar, Kisumu in Kenya. Approximate $4 \%$ were from tropical/subtropical highland climate areas such as Nairobi in Kenya, the Lijiang National Park, and the Potatso National Park in China. A small portion (2.1\%) was from the cold areas, such as Jiuzhaigou National Park in China, and Alaska in the 
239 USA (Fig 1A). We collected the samples from different landforms, including hills, mountains,

240 plateaus, canyons, valleys, and bays. Notably, some fungi were isolated from samples

241 collected from the mountains with an altitude over 3,000 meters, such as the Cang Mountains

242 and Meili Snow Mountains in the Yunnan Province, China. The vast difference of sample

243 collection in location, weather, climate, and altitude (Fig 1B) promises the diversity of fungal

244 species and their genetic background.

245 To maximize the diversity and quantity in our fungal library, we selected fungal colonies

246 based on their location, color, and morphology on culture plates. Therefore, the fungal isolates

247 in the library look strikingly different (Fig 1C). This visible criterion facilitates the fungal library

248 construction. However, we also discarded many fungi that are different species with similar

249 morphology. We examined the species diversity of our fungal library at the molecular level by

250 randomly picking 40 fungal isolates. Their genomic DNA was isolated, the ITS regions were

251 amplified, and PCR products were sequenced. These sequences have been deposited into

252 GenBank at https://www.ncbi.nlm.nih.gov/genbank/sequenceids/. Their Accession numbers are

MT594355-MT594393 and MT584204. We searched these sequences against NCBI DNA

254

databases using blast. Results show that about $12.5 \%$ of total fungal isolates have identical ITS

255

sequences to others in the library (Table 3). For instance, 126-G10, 117-B9, and 45-F10 have

256

identical ITS to Fusarium solani, and 2 of 3 might be duplicates. Stains 99-H5 and 78-D10 have

257

identical ITS to Penicillium sclerotiorum. More than $80 \%$ of fungal isolates belong to different

258

species or strains, indicating the fungal library is highly diverse. A small portion $(<12.5 \%)$ are

259

duplications of the other. Based on these ITS sequences, a phylogenetic tree was constructed,

displaying the fungal diversity in samples (Supplemental File 1). Three genera (Trichoderma,

261 Fusarium and Penicillium) present in three big branches, which is consistent to our sampling

262 sources, e.g., soil and plants. Trichoderma and Fusarium are the most prevalent soil fungi and

263 many are associated with plants (Harman, Howell et al. 2004). Penicillium is ubiquitous genus

264 with more than 350 species already identified (Visagie, Houbraken et al. 2014).

PeerJ reviewing PDF | (2020:05:49300:2:1:NEW 24 Oct 2020) 
265 Endophytic fungi from Chinese medicinal plants

266

267

268

269

270

271

272

273

274

275

276

277

278

279

280

281

282

283

284

285

286

287

288

289

290

Our fungal library includes many endophytic fungi. Since endophytic fungi produce many plant metabolites with medical functions, we collected plant samples from the three most extensive tropical botanic gardens in China, including South China, Hainan Xinglong, and Yunnan Tropical. There are many diverse plants in these gardens. We collected 27 well-known Chinese medicinal plants, such as Chinese black olive (Canarium pimela), Chinese croton (Excoecaria cochinchinensis), Lemon-scented gum (Eucalyptus citriodora Hook), Sweet osmanthus (Osmanthus fragrans), and Yellow cow wood (Cratoxylum cochinchinense) (Table 4). We separated different parts from each plant, e.g., leaf, bark, stem, fruits, and seeds, and sterilized their surfaces with $75 \%$ ethanol. Following grinding masses and culturing on Malt Extract Agar (MEA) plates, more than 50 endophytic fungi were isolated on MEA plates. The colors and morphology of these endophytic fungi and their corresponding plants look strikingly different (Fig 2). The ITS of three fungi were PCR-amplified and sequenced (Accession \# in GenBank are MT994711, MT994712, and MT594489). They were identified as Stephanonectria keithii (Fig 2C), Aspergillus sp. (Fig 2E), and Tolypocladium album (Fig 2Z), respectively.

\section{Construction of a fungal extract library}

To generate a fungal metabolite library, we used the cereal-based medium to produce the secondary metabolites as reported (Niu, Wang et al. 2015). Each fungus was cultured in a testing tube with six small pieces of cereals. After culturing for one month, we used ethyl acetate to extract the secondary metabolites. We obtained 9,053 crude extracts in total, each of which corresponds to a specific fungal isolate. As anticipated, different fungal strains produced different amounts of secondary metabolites from $1 \mathrm{mg}$ to $20 \mathrm{mg}$ per gram culture, and have various physical features such as stickiness, odors, and solubility. More than $90 \%$ of the extracts have colors, including green, orange, red, yellow, purple, and others. The crude extracts were dissolved in DMSO to generate $2 \mathrm{mg} / \mathrm{mL}$ solution. For future reference, we name this library "Global Fungal Extract Library" or GFEL in brief. 
291

292

293

294

295

296

297

298

299

300

301

302

303

304

305

306

307

308

309

310

311

312

313

314

315

316

\section{Screen the fungal extract library against $P$. falciparum transmission to mosquitoes}

Malaria remains a devastating disease, and Anopheles midgut protein fibrinogen-related protein 1 (FREP1) mediates Plasmodium transmission (Li, Wang et al. 2013, Zhang, Niu et al. 2015, Niu, Franca et al. 2017). FREP1 mediates Plasmodium invasion in mosquitoes by binding to $P$. falciparum gametocytes or ookinetes (Zhang, Niu et al. 2015).

To examine the usability of the newly constructed fungal library, we screened 460 fungal extracts obtained in later 2016 and early 2017 for their inhibition activity against FREP1-P. falciparum interaction and found 4 extracts that prevented FREP1 from binding to $P$. falciparum lysates by over $90 \%$. The fungal colonies of these four fungal candidates on MEA plates showed different colors and shapes (Fig 3A-D). Then, we determined the activities of the four candidates against $P$. falciparum to An. gambiae in vivo using the standard membrane feeding assays (SMFA). Results show that the fungal extracts of $37 \mathrm{C} 6$ and $22 \mathrm{E} 8$ could block malaria transmission at a concentration of $100 \mu \mathrm{g} / \mathrm{mL}$, and 100D3 and 45F10 did not (Fig 3E,

Supplemental File 2). The oocyst numbers of $37 \mathrm{C} 6$ or 22E8 extract-treated mosquitoes were nearly zero, while the oocyst numbers in the 100D3 or 45F10 extract-treated mosquitoes were not significantly different from that of the DMSO control (Fig. 3E). After further dilution of the two positive candidate fungal extracts (37C6 and 22E8) to $20 \mu \mathrm{g} / \mathrm{mL}$, we found that the extract of 22E8 still significantly inhibited the activity in $P$. falciparum transmission to mosquitoes (Fig 3 F, Supplemental File 2). We sequenced the ITS sequences of the four candidate fungi and their accession number are MT594486-MT594488 and MT613342 at GenBank at https://www.ncbi.nlm.nih.gov/genbank/sequenceids/. According to ITS sequences of 22E8 (Acc \#: MT613342) and 37C6 (Acc\#: MT594487), the fungal species of 22E8 and 37C6 were Penicillium thomii and Penicillium pancosmium, respectively (Table 5). Notably, this is the first report about $P$. thomii and $P$. pancosmium that produce secondary metabolites with antimalarial activities. An independent project in our lab identified Asperaculane $B$ as an active compound from this GFEL that inhibited malaria transmission to mosquitoes (Niu, Hao et al. 2020).

Peer] reviewing PDF | (2020:05:49300:2:1:NEW 24 Oct 2020) 
317 Examine the usability of fungal extract library in finding antibiotic leads

318 Antibiotic-resistant bacteria threaten public health (Todd 2017). We screened potential

319 antibiotics from this newly established fungal library against antibiotic-resistant bacterial

320 pathogens. We randomly picked 574 fungal extracts and examined their activity in inhibiting

321 Gram-positive (methicillin-resistant S. aureus MRSA) and Gram-negative (S. flexneri) bacterial

322 pathogens. These fungi were isolated from samples collected in late 2017. Among them, 47

323 inhibited the growth of S. aureus MRSA (hit rate of $10.8 \%$ ), and one inhibited the growth of $S$.

324 flexneri (hit rate of $0.17 \%$, Table 6). The hit rate of antibiotics against Gram-positive bacteria

325 was higher than that of the Gram-negative bacteria, which consistent to the well-known

326 challenges in antibiotic discovery against gram-negative pathogens. Gram-negative pathogens

327 have unique outer membrane and efflux pumps (Fair and Tor 2014).

328 We also examined the effect of 288 fungal extracts on non-methicillin-resistant $S$.

329 aureus. The results showed that 22 prevented the growth of non-methicillin-resistant $S$. aureus

330 (hit rate of 7.6\%). Besides, we analyzed another two featured bacteria, Mycobacterium

331 smegmatis, which is gram-positive and acid-fast dye staining cell wall, and E. coli-AS17tolc,

332 which has gram-negative cell wall, but more permeable than the wild type E. coli. We obtained

333 eight candidates against Mycobacterium smegmatis and three candidates against E. coli-

334 AS17tolc. The results showed $10.8 \%$ and $1 \%$ hit rates to Mycobacterium smegmatis and E. coli-

335 AS17tolc, respectively (Table 6 and Supplemental File 3). The results show that the secondary

336 metabolites produced by different types of fungi in the GFEL contained compounds biologically

337 against multi-resistant, various pathogenic strains of bacteria.

338 Examine the usability of fungal extract library in finding anti-chronic myeloid leukemia

339 candidates

$340 \quad$ Finally, we studied the possibility of obtaining drug candidate leads against chronic

341 myelocytic leukemia (CML), a malignant tumor of the blood system (Kaleem, Shahab et al.

342 2015). A small subset of endophytic fungi from Chinese medicinal plants was used for this

Peer) reviewing PDF | (2020:05:49300:2:1:NEW 24 Oct 2020) 
343 purpose. Fifty extracts were examined for their inhibition against the human immortalized

344 myelogenous leukemia cell line K562 using the MTT method. The final concentrations of crude

345 extracts were $20 \mu \mathrm{g} / \mathrm{mL}$. The results from triplicates showed that 4 extracts $(\# 17, \# 22, \# 29$, \#35)

346 significantly inhibited the proliferation of K562 cells ( $p<0.05$, Fig 4, Supplemental File 4). The

347 hit rate of the plant-fungal metabolite library was $8 \%$. Notably, the survivorship of K562 cells

348 with extract \#29 was about $24.8 \%$ compared with the control, e.g., the inhibition of extract \#29

349 on K562 proliferation was as high as $75.2 \%$. The specimen of \#29 was from the fungus (Fig 2Z)

350 isolated from the plant Litsea glutinosa (Fig 2HH) collected from the Medical Botanical Garden

351 of South China Botanical Garden in Guangzhou. Litsea glutinosa has been used to treat

352 diarrhea, traumatic injuries, mumps, and rheumatism. It contains abundant flavonoids, terpenes,

353 and alkaloids, and has good antibacterial activity, immunomodulatory effect, and anti-tumor

354 effect. We PCR-amplified the ITS region of the fungal candidate (\#29) and sequenced the

355 region. Based on the ITS region sequence (Accession \# at GenBank: MT594489), the candidate

356 fungus was Tolypocladium album. Notably, a tetrameric acid from Tolypocladium album has

357 been reported to inhibit the tumor's growth (Fukuda, Sudoh et al. 2015). Further studies will be

358 conducted to isolate and identify the bioactive compounds from this fungus.

359

360

\section{Conclusions}

361 We established a comprehensive fungal library that is diverse and useful for communities. We

362 also demonstrated the usability of GFEL and identified a set of fungal strains that produce

363 secondary metabolites to inhibit Plasmodium falciparum's transmission, chronic pneumonia development, and bacteria proliferation. Further studies will identify the active compounds for 365 drug development.

366

\section{Acknowledgments}


368 We thank numerous residents and colleagues for collecting samples from various places and 369 mailing the samples to us. We also appreciated many undergraduate students and technicians 370 in culturing and isolating fungi.

371

\section{References}

373 Blackwell, M. (2011). "The fungi: 1, 2, 3 ... 5.1 million species?" Am J Bot 98(3): 426-438.

374 Buyel, J. F. (2018). "Plants as sources of natural and recombinant anti-cancer agents."

375 Biotechnology Advances 36(2): 506-520.

376 Dias, D. A., S. Urban and U. Roessner (2012). "A historical overview of natural products in drug

377 discovery." Metabolites 2(2): 303-336.

378 Elder, A. L. (1944). "Fungi for Penicillin Production." Science 99(2563): 119-120.

379 Fair, R. J. and Y. Tor (2014). "Antibiotics and bacterial resistance in the 21st century." Perspect 380 Medicin Chem 6: 25-64.

381 Fleming, A. (1929). "On the antibacterial action of cultures of a Penicillium with special

382 reference to their use in the isolation of $\mathrm{B}$. influenza." Br J Exp Pathol. 10: 11.

383 Fukuda, T., Y. Sudoh, Y. Tsuchiya, T. Okuda, N. Matsuura, A. Motojima, T. Oikawa and Y. Igarashi (2015). "Tolypoalbin, a new tetramic acid from Tolypocladium album TAMA 479." $\underline{\mathrm{J}}$ Antibiot (Tokyo) 68(6): 399-402.

Gonzalez-Menendez, V., G. Crespo, N. de Pedro, C. Diaz, J. Martin, R. Serrano, T. A. Mackenzie, C. Justicia, M. R. Gonzalez-Tejero, M. Casares, F. Vicente, F. Reyes, J. R. Tormo and O. Genilloud (2018). "Fungal endophytes from arid areas of Andalusia: high potential sources for antifungal and antitumoral agents." Sci Rep 8(1): 9729.

390 Harman, G. E., C. R. Howell, A. Viterbo, I. Chet and M. Lorito (2004). "Trichoderma species-opportunistic, avirulent plant symbionts." Nat Rev Microbiol 2(1): 43-56.

392 Hawksworth, D. L. and R. Lucking (2017). "Fungal Diversity Revisited: 2.2 to 3.8 Million

393 Species." Microbiol Spectr 5(4).

394 Higgins, D. G. and P. M. Sharp (1988). "CLUSTAL: a package for performing multiple sequence 395 alignment on a microcomputer." Gene 73(1): 237-244.

396 Hsu, E. (2006). "The history of qing hao in the Chinese materia medica." Trans R Soc Trop Med 397 Hyg 100(6): 505-508.

398 Hussain, H., A. M. Al-Sadi, B. Schulz, M. Steinert, A. Khan, I. R. Green and I. Ahmed (2017). "A 399 fruitful decade for fungal polyketides from 2007 to 2016: antimicrobial activity, chemotaxonomy 400 and chemodiversity." Future Med Chem 9(14): 1631-1648. 
401 Kaleem, B., S. Shahab, N. Ahmed and T. S. Shamsi (2015). "Chronic Myeloid Leukemia--

402 Prognostic Value of Mutations." Asian Pac J Cancer Prev 16(17): 7415-7423.

403 Keller, N. P. (2019). "Fungal secondary metabolism: regulation, function and drug discovery." 404 Nat Rev Microbiol 17(3): 167-180.

405 Lenzi, J., T. M. Costa, M. D. Alberton, J. A. G. Goulart and L. B. B. Tavares (2018). "Medicinal 406 fungi: a source of antiparasitic secondary metabolites." Appl Microbiol Biotechnol.

407 Li, H. Y., C. Qing, Y. L. Zhang and Z. W. Zhao (2005). "Screening for endophytic fungi with 408 antitumour and antifungal activities from Chinese medicinal plants." World Journal of 409 Microbiology \& Biotechnology 21(8-9): 1515-1519.

410 Li, J., X. Wang, G. Zhang, J. Githure, G. Yan and A. A. James (2013). "Genome-block 411

412

413 expression-assisted association studies discover malaria resistance genes in Anopheles gambiae." Proceedings of the National Academy of Sciences of the United States of America 110(5): 20675-20680.

414 Luo, X. D. and C. C. Shen (1987). "The Chemistry, Pharmacology, and Clinical-Applications of 415 Qinghaosu (Artemisinin) and Its Derivatives." Medicinal Research Reviews 7(1): 29-52.

416 May, T., J. Milne, A. Wood, S. Shingles, R. Jones and P. Nwiah (2004). Interactive Catalogue of 417 Australian Fungi. C. R. B. Gardens. Melbourne, Australian Biological Resources Study.

418 Nielsen, J. C. and J. Nielsen (2017). "Development of fungal cell factories for the production of 419 secondary metabolites: Linking genomics and metabolism." Synth Syst Biotechnol 2(1): 5-12.

420 Niu, G., A. C. Franca, G. Zhang, W. Roobsoong, W. Nguitragool, X. Wang, J. Prachumsri, N. S. 421 Butler and J. Li (2017). "The fibrinogen-like domain of FREP1 protein is a broad-spectrum 422 malaria transmission-blocking vaccine antigen." J Biol Chem 292(28): 11960-11969.

423 Niu, G., Y. Hao, X. Wang, J. M. Gao and J. Li (2020). "Fungal Metabolite Asperaculane B 424 Inhibits Malaria Infection and Transmission." Molecules 25(13).

425 Niu, G., B. Wang, G. Zhang, J. B. King, R. H. Cichewicz and J. Li (2015). "Targeting mosquito 426 FREP1 with a fungal metabolite blocks malaria transmission." Sci Rep 5: 14694.

427 O'Brien, J., I. Wilson, T. Orton and F. Pognan (2000). "Investigation of the Alamar Blue 428 (resazurin) fluorescent dye for the assessment of mammalian cell cytotoxicity." Eur J Biochem 429 267(17): 5421-5426.

430 Op De Beeck, M., B. Lievens, P. Busschaert, S. Declerck, J. Vangronsveld and J. V. Colpaert 431 (2014). "Comparison and validation of some ITS primer pairs useful for fungal metabarcoding 432 studies." PLoS One 9(6): e97629.

433 Pham, J. V., M. A. Yilma, A. Feliz, M. T. Majid, N. Maffetone, J. R. Walker, E. Kim, H. J. Cho, J. 434 M. Reynolds, M. C. Song, S. R. Park and Y. J. Yoon (2019). "A Review of the Microbial 435 Production of Bioactive Natural Products and Biologics." Front Microbiol 10: 1404. 
436 Raja, H. A., A. N. Miller, C. J. Pearce and N. H. Oberlies (2017). "Fungal Identification Using 437 Molecular Tools: A Primer for the Natural Products Research Community." J Nat Prod 80(3): 438 756-770.

439 Richards, T. A., M. D. Jones, G. Leonard and D. Bass (2012). "Marine fungi: their ecology and 440 molecular diversity." Ann Rev Mar Sci 4: 495-522.

441 Todd, B. (2017). "Reconsidering Antibiotic Resistance." Am J Nurs 117(12): 66-67.

442 van Dijk, J. W. and C. C. Wang (2016). "Heterologous Expression of Fungal Secondary 443 Metabolite Pathways in the Aspergillus nidulans Host System." Methods Enzymol 575: 127-142.

444 Venieraki, A., M. Dimou and P. Katinakis (2017). "Endophytic fungi residing in medicinal plants 445 have the ability to produce the same or similar pharmacologically active secondary metabolites 446 as their hosts." Hellenic Plant Protection Journal 10(2): 16.

447 Visagie, C. M., J. Houbraken, J. C. Frisvad, S. B. Hong, C. H. Klaassen, G. Perrone, K. A. 448 Seifert, J. Varga, T. Yaguchi and R. A. Samson (2014). "Identification and nomenclature of the 449 genus Penicillium." Stud Mycol 78: 343-371.

450 Wolfender, J. L. and E. F. Queiroz (2012). "New Approaches for Studying the Chemical 451 Diversity of Natural Resources and the Bioactivity of their Constituents." Chimia 66(5): 324-329.

452 Zhang, G., G. Niu, C. M. Franca, Y. Dong, X. Wang, N. S. Butler, G. Dimopoulos and J. Li 453 (2015). "Anopheles Midgut FREP1 Mediates Plasmodium Invasion." J Biol Chem 290(27): 454 16490-16501.

455 Zhang, X., S. J. Li, J. J. Li, Z. Z. Liang and C. Q. Zhao (2018). "Novel Natural Products from 456 Extremophilic Fungi." Mar Drugs 16(6).

457 Zhang, X. Y., G. L. Tang, X. Y. Xu, X. H. Nong and S. H. Qi (2014). "Insights into deep-sea 458 sediment fungal communities from the East Indian Ocean using targeted environmental 459 sequencing combined with traditional cultivation." PLoS One 9(10): e109118. 


\section{Figure 1}

Worldwide localization of samples, distribution of collected fungi in different climate zones, and some fungal morphology.

(A): Samples were collected worldwide, pointed by red dots. (B): Distribution of the collected fungi in different climates zones. The climate classification was based on the Köppen-Geiger Climate Classification. The codes of the climate are as the following: Aw: Tropical monsoon climate; Bsk: Cold semi-arid climate; Cfa: Humid subtropical climate; Cfb: Temperate oceanic climate; Cwa: Monsoon-influenced humid subtropical climate; Cwb: Subtropical highland climate or Monsoon-influenced temperate marine climate; Dfb: Humid continental climate; Dwa: Monsoon-influenced hot-summer humid continental climate; Dwb: Monsoon-influenced warm-summer humid continental climate. (C): Morphology of some isolated fungal colonies in the library. 

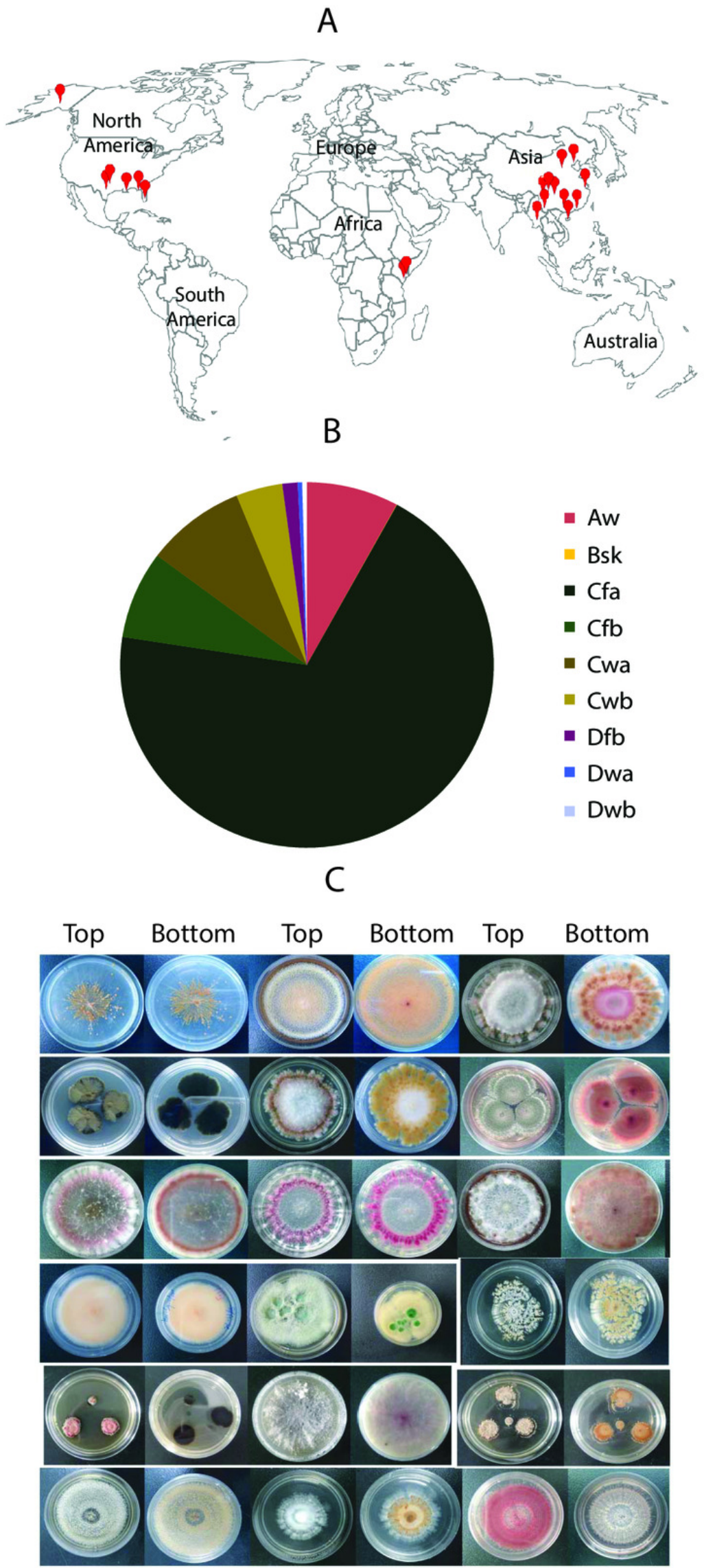

Peer) reviewing PDF | (2020:05:49300:2:1:NEW 24 Oct 2020) 


\section{Figure 2}

The fungal isolates and the corresponding Chinese medicinal host plants.

The images in rows 1,3 , and 5 were the fugal isolates grown on MEA plates, and the photos below in row 2, 4, and 6 are the corresponding host plants where they were isolated.

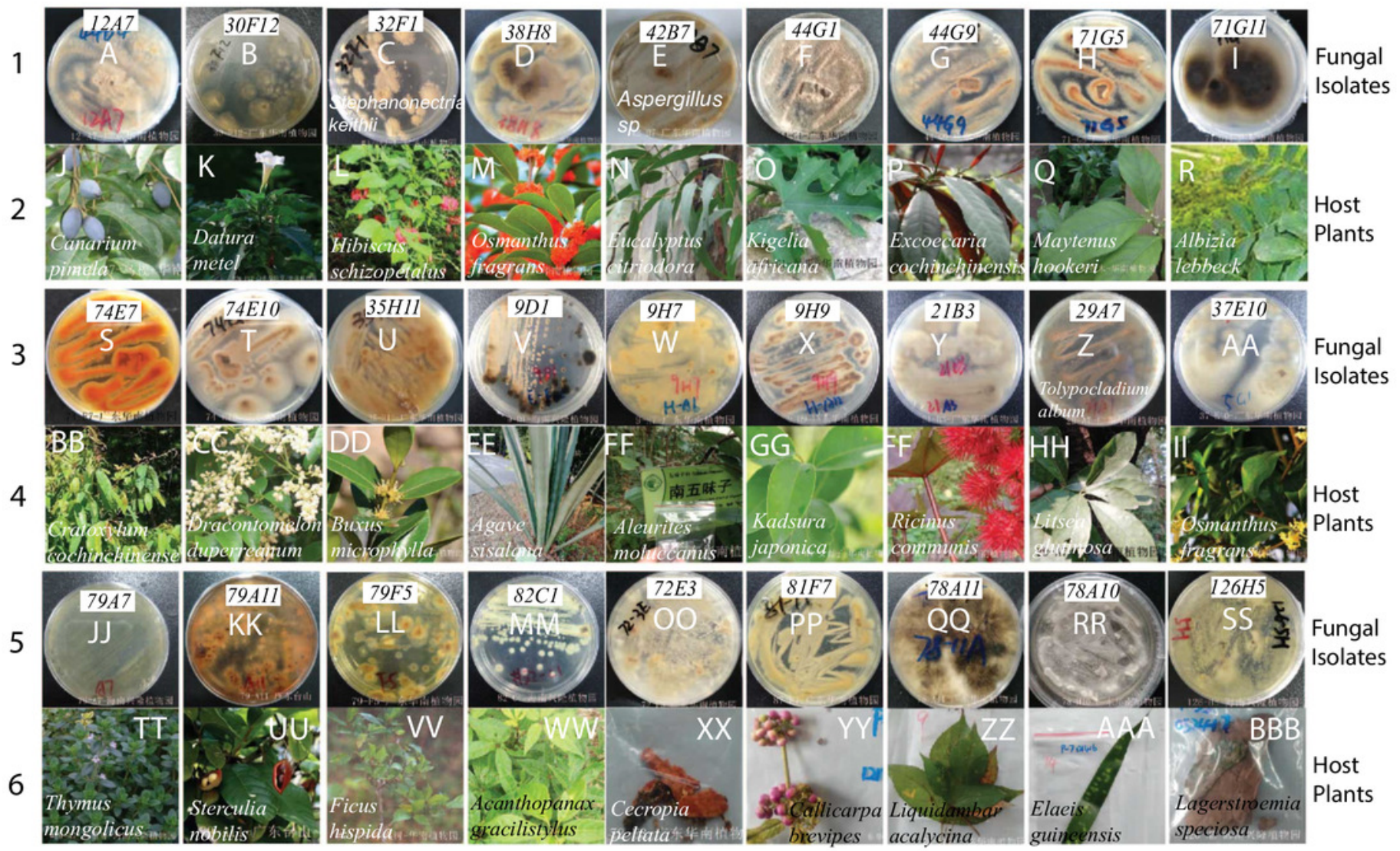




\section{Figure 3}

The transmission-blocking activity of the extracts candidates by screening the fungal library with the in vitro FREP1-parasite interaction-based ELISA assays.

(A-D): The morphology of fungal isolates 37C6, 100D3, 22E8T, and 45F10 on the MEA agar plate. (E): The final concentrations of the fungal extracts were $100 \mu \mathrm{g} / \mathrm{mL}$ and the results show that the fungal extracts (37C6 and 22E8) significantly reduced the oocyst number compared with the DMSO control while the oocyst number of the other two (100D3 and 45F10) was not significantly different with the DMSO control, respectively. (F): Further, the fungal extract of 37C5 continued to show a significant reduction of the oocyst number in midgut while the 22E8 fungal extract did not have significant effects on $P$. falciparum infection in mosquitoes when the concentration of the fungal extracts was decreased to 20 $\mu \mathrm{g} / \mathrm{mL}$. $\mathbf{N}$ : the number of mosquitoes for each treatment; mean: the average number of oocysts per midgut; PR: infection prevalence in mosquitoes. $\boldsymbol{p}$ : the $\mathbf{p}$-value was calculated by the Mann-Whitney-Wilcoxon test. The experiments were repeated three times. 

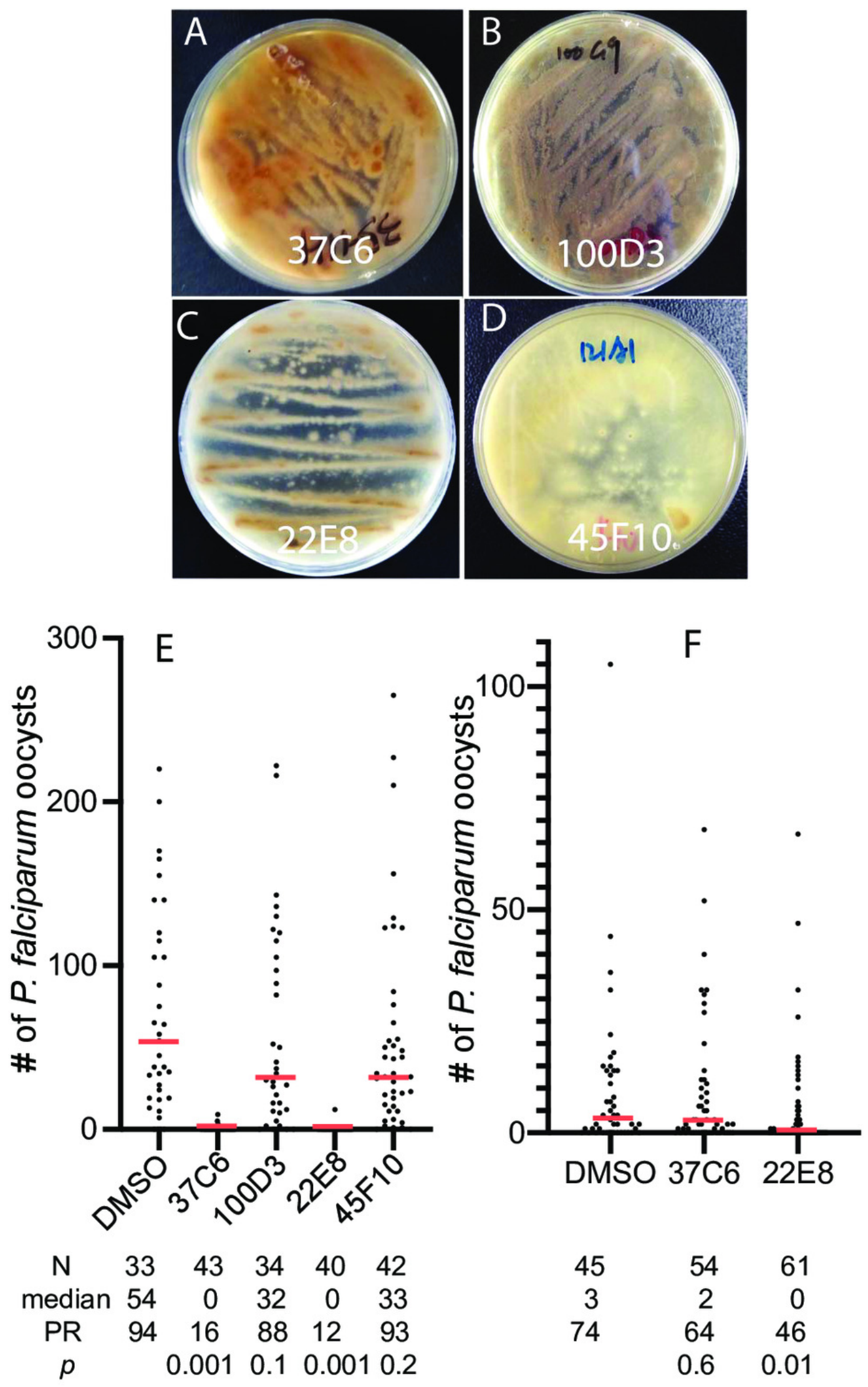


\section{Figure 4}

Anti-chronic myeloid leukemia screening results.

About 50 extracts of endophytic fungi isolated from the Chinese medicinal plant-fungal metabolite library were examined against K562 cells using the MTT method. The results show that 4 extracts (\#17, \#22, \#29, \#35) significantly inhibited the growth of K562 cells ( $p$ $<0.05)$. Notably, the survivorship of K562 cells with extract \#29 was about $24.3 \%$ compared with the control, or the inhibition of extract \#29 on K562 was 75.7\%. The DMSO was applied as the control. The data shows the means and standard deviations of triplicates.

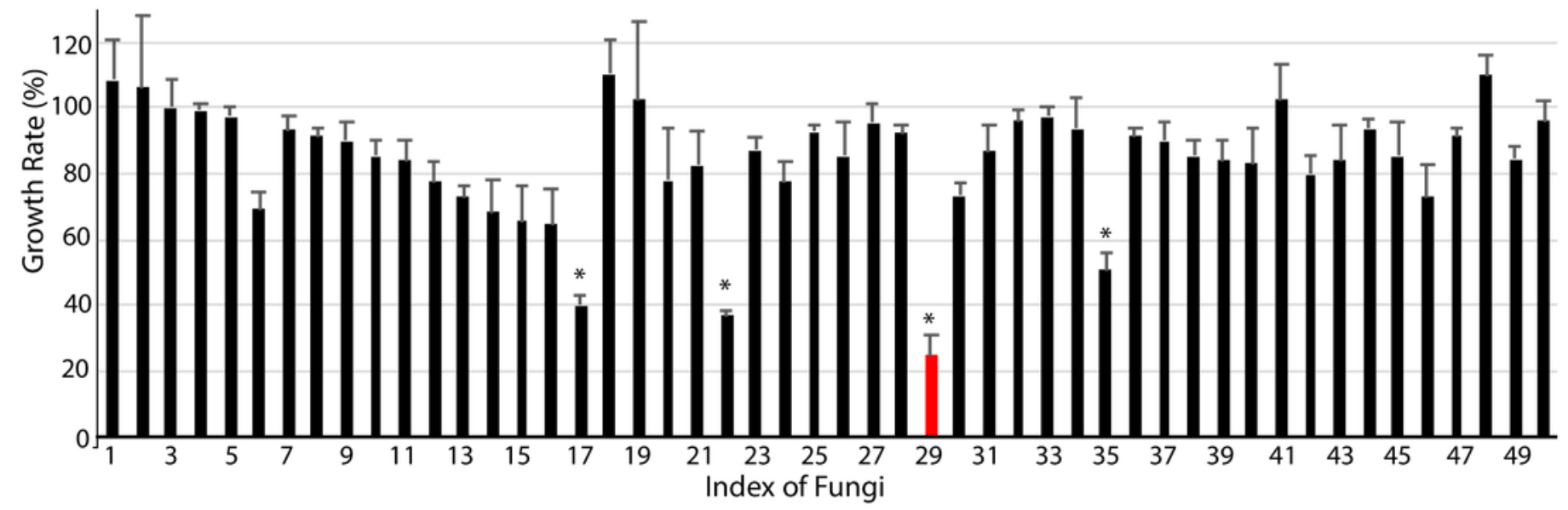




\section{Table 1 (on next page)}

Table 1: PCR Primers for fungal ITS regions 
1

\begin{tabular}{|l|l|l|}
\hline Primer name & sequences & rDNA region \\
\hline ITS1F $(\mathrm{F})$ & CTTGGTCATTTAGAGGAAGTAA & $18 \mathrm{~S}$ \\
\hline ITS2 $(\mathrm{R})$ & GCTGCGTTCTTCATCGATGC & $5.8 \mathrm{~S}$ \\
\hline ITS3 $(\mathrm{F})$ & GCATCGATGAAGAACGCAGC & $5.8 \mathrm{~S}$ \\
\hline ITS4 (R) & TCCTCCGCTTATTGATATGC & $28 \mathrm{~S}$ \\
\hline ITS86F (F) & GTGAATCATCGAATCTTTGAA & $5.8 \mathrm{~S}$ \\
\hline ITS86R (R) & TTCAAAGATTCGATGATTCAC & $5.8 \mathrm{~S}$ \\
\hline
\end{tabular}

2

3

4 
Table 2 (on next page)

Fungal strains collected from different locations of the world 


\begin{tabular}{|c|c|c|c|c|}
\hline \# of isolates & Area/City/County & State/Province & Country & Continent \\
\hline 188 & Norman & Oklahoma & USA & North America \\
\hline 287 & Oklahoma City & Oklahoma & USA & North America \\
\hline 208 & Stillwater & Oklahoma & USA & North America \\
\hline 76 & Dallas & Texas & USA & North America \\
\hline 68 & New Orleans & Louisiana & USA & North America \\
\hline 59 & Pensacola & Florida & USA & North America \\
\hline 47 & Tallahassee & Florida & USA & North America \\
\hline 33 & Lake City & Florida & USA & North America \\
\hline 401 & Miami & Florida & USA & North America \\
\hline 119 & Juneau & Alaska & USA & North America \\
\hline 702 & Nairobi & NA & Kenya & Africa \\
\hline 322 & Yangon & NA & Myanmar & Asia \\
\hline 181 & Shanghai & NA & China & Asia \\
\hline 34 & Beijing & NA & China & Asia \\
\hline 39 & Chongqing & NA & China & Asia \\
\hline 1469 & Foshan & Guangdong & China & Asia \\
\hline 472 & Guangzhou & Guangdong & China & Asia \\
\hline 1093 & South China & Guangdong & China & Asia \\
\hline & Botanical Garden & & & \\
\hline 667 & Taishan & Guangdong & China & Asia \\
\hline 37 & Zhongshan & Guangdong & China & Asia \\
\hline 45 & Jiangmen & Guangdong & China & Asia \\
\hline 14 & Maoming & Guangdong & China & Asia \\
\hline 351 & Shaoguan & Guangdong & China & Asia \\
\hline 34 & Shenzhen & Guangdong & China & Asia \\
\hline 356 & Laibin & Guangxi & China & Asia \\
\hline 54 & Liuzhou & Guangxi & China & Asia \\
\hline 39 & Rongan & Guangxi & China & Asia \\
\hline 808 & Zhongshan & Guangxi & China & Asia \\
\hline 46 & Qinzhou & Guangxi & China & Asia \\
\hline 135 & Lijiang & Yunnan & China & Asia \\
\hline 94 & Cang Mountain & Yunnan & China & Asia \\
\hline 254 & Xishuangbanna & Yunnan & China & Asia \\
\hline & $\begin{array}{c}\text { Tropical Botanical } \\
\text { Garden }\end{array}$ & & & \\
\hline 77 & Potatso National Park & Yunnan & China & Asia \\
\hline 50 & Tongzi & Guizhou & China & Asia \\
\hline 38 & $\begin{array}{c}\text { Jiuzhaigou National } \\
\text { Park }\end{array}$ & Sichuan & China & Asia \\
\hline 132 & $\begin{array}{l}\text { Xinglong Tropical } \\
\text { Botanical Garden }\end{array}$ & Hainan & China & Asia \\
\hline 3 & Hohhot & Inner Mongolia & China & Asia \\
\hline 21 & Changzhi & Shanxi & China & Asia \\
\hline
\end{tabular}


Table 3 (on next page)

The species of randomly sampled fungi from the library 


\begin{tabular}{|c|c|c|c|c|c|c|}
\hline ID & Species & Length & Coverage & Identity & Source & Sample location \\
\hline 11-A5 & Acremonium cellulolyticus & 581 & $100 \%$ & $100 \%$ & soil & Guangxi, China \\
\hline $\mathbf{S 3} / \mathbf{2}$ & Albifimbria verrucaria & 582 & $98 \%$ & $100 \%$ & soil & Guangdong, China \\
\hline 116-E12 & Arthropsis hispanica & 455 & $100 \%$ & $100 \%$ & soil & Florida, USA \\
\hline 4-H9 & Ascomycota sp. & 586 & $100 \%$ & $100 \%$ & plant & Guangdong, China \\
\hline 64-A1 & Aspergillus flavus & 597 & $100 \%$ & $100 \%$ & plant & Oklahoma, USA \\
\hline 79-B7 & Aspergillus sp. & 574 & $100 \%$ & $100 \%$ & soil & Guangdong, China \\
\hline 49-G11 & Aspergillus sydowii & 569 & $100 \%$ & $100 \%$ & air & Guangdong, China \\
\hline 58-C1 & Aspergillus sp. & 621 & $92 \%$ & $77 \%$ & soil & Guangdong, China \\
\hline 3-D7 & Cylindrocladium sp. & 575 & $100 \%$ & $99 \%$ & soil & Guangdong, China \\
\hline 24-C5 & Debaryomyces subglobosus & 634 & $100 \%$ & $100 \%$ & soil & Guangdong, China \\
\hline 116-H3 & Epicoccum sorghinum & 589 & $100 \%$ & $100 \%$ & plant & Guangxi, China \\
\hline 88-E10 & Fusarium kyushuense & 540 & $95 \%$ & $100 \%$ & soil & Guangdong, China \\
\hline 126-G10 & Fusarium solani & 573 & $100 \%$ & $100 \%$ & soil & Guangxi, China \\
\hline 117-B9 & Fusarium solani & 576 & $100 \%$ & $100 \%$ & soil & Florida, USA \\
\hline 45-F10 & Fusarium solani & 573 & $100 \%$ & $100 \%$ & soil & Guangdong, China \\
\hline 3-F5 & Fusarium verticillioides & 562 & $100 \%$ & $100 \%$ & soil & Guangxi, China \\
\hline 18-F5 & Trichoderma sp. & 619 & $99 \%$ & $92 \%$ & plant & Hainan, China \\
\hline 66-B4 & Metarhizium sp. & 617 & $100 \%$ & $99 \%$ & soil & Yunnan, China \\
\hline 17-A1 & Mycosphaerella sp. & 573 & $100 \%$ & $100 \%$ & soil & Alaska, USA \\
\hline HW & Neurospora sp. & 586 & $100 \%$ & $99 \%$ & air & Guangdong, China \\
\hline 74-F11 & Penicillium rolfsii & 591 & $99 \%$ & $100 \%$ & soil & Guangdong, China \\
\hline 99-H4 & Penicillium sclerotiorum & 586 & $100 \%$ & $100 \%$ & plant & Guangxi, China \\
\hline 78-D10 & Penicillium sclerotiorum & 583 & $100 \%$ & $100 \%$ & soil & Guangdong, China \\
\hline 81-D8 & Penicillium sp. & 575 & $100 \%$ & $99 \%$ & soil & Guangdong, China \\
\hline 107-H3 & Penicillium soppii & 470 & $100 \%$ & $100 \%$ & soil & Alaska, USA \\
\hline 125-B10 & Penicillium sp. & 589 & $100 \%$ & $100 \%$ & soil & Guangdong, China \\
\hline 114-D12 & Penicillium sp. & 584 & $100 \%$ & $100 \%$ & plant & Yangon, Myanmer \\
\hline 3-G10 & Talaromyces stipitatus & 582 & $100 \%$ & $100 \%$ & soil & Guangxi, China \\
\hline 37-A6 & Trichoderma sp. & 612 & $83 \%$ & $86 \%$ & plant & Guangdong, China \\
\hline 95-C6 & Trichoderma sp. & 571 & $100 \%$ & $99 \%$ & soil & Guangxi, China \\
\hline 45-G11 & Trichoderma atroviride & 629 & $100 \%$ & $100 \%$ & soil & Guangdong, China \\
\hline CL & Trichoderma sp. & 612 & $100 \%$ & $99 \%$ & soil & Maasi Marla, Kenya \\
\hline 16-D5 & Trichoderma harzianum & 625 & $100 \%$ & $100 \%$ & soil & Guangdong, China \\
\hline 100-D3 & Trichoderma sp. & 554 & $100 \%$ & $99 \%$ & soil & Guangdong, China \\
\hline 104-D12 & Trichoderma harzianum & 623 & $100 \%$ & $100 \%$ & soil & Guangxi, China \\
\hline 98-C8 & Trichoderma sp. & 610 & $100 \%$ & $99 \%$ & soil & Guangxi, China \\
\hline 80-G9 & Trichoderma sp. & 622 & $100 \%$ & $99 \%$ & soil & Guangdong, China \\
\hline 114-G8 & Trichoderma sp. & 617 & $100 \%$ & $99 \%$ & soil & Guangdong, China \\
\hline 117-H9 & Trichoderma harzianum & 624 & $100 \%$ & $100 \%$ & soil & Florida, USA \\
\hline 121-G6 & Metarhizium carneum & 172 & $100 \%$ & $100 \%$ & plant & Hainan, China \\
\hline
\end{tabular}




\section{Table 4 (on next page)}

Some fungal strains isolated from Chinese medicinal plants. 


\begin{tabular}{|c|c|c|c|c|}
\hline ID & Sample collection location & Medical plant/tissue & Family & Species \\
\hline $12 \mathrm{~A} 7$ & SCBG, Guangzhou, China & Chinese black olive/stem & Burseraceae & Canarium pimela \\
\hline 30F12 & SCBG, Guanzhou, China & Devil's Trumpet/leaf & Solanaceae & Datura metel \\
\hline 32F1 & SCBG, Guanzhou, China & Japanese lantern & Malvaceae & Hibiscus schizopetalus \\
\hline $38 \mathrm{H8}$ & SCBG, Guangzhou, China & Sweet osmanthus/leaf & Oleaceae & Osmanthus fragrans (Thunb) \\
\hline 42B7 & SCBG, Guangzhou, China & Lemon-scented gum/bark & Myrtaceae & Eucalyptus citriodora Hook.f \\
\hline 44G1 & SCBG, Guangzhou, China & Sausage Tree/leaf & Bignoniaceae & Kigelia africana (Lam.) Benth. \\
\hline 44G9 & SCBG, Guangzhou, China & Chinese croton/leaf & Euphorbiaceae & Excoecaria cochinchinensis \\
\hline $71 G 5$ & SCBG, Guangzhou, China & Maytenus hookeri Loes/leaf & Celastraceae & Maytenus hookeri Loes. \\
\hline $71 G 11$ & SCBG, Guangzhou, China & Siris tree/leaf & Fabaceae & Albizia lebbeck \\
\hline 74E7 & SCBG, Guangzhou, China & Yellow cow wood/leaf & Hypericaceae & Cratoxylum cochinchinense \\
\hline 74E10 & SCBG, Guangzhou, China & $\begin{array}{c}\text { Dracontomelon } \\
\text { duperreanum/leaf }\end{array}$ & Anacardiaceae & Dracontomelon duperreanum \\
\hline $35 \mathrm{H11}$ & SCBG, Guangzhou, China & Harland boxwood/leaf & Buxaceae & Buxus microphylla \\
\hline 9D1 & HXTBG, Haikou, China & Sisal/leaf & Asparagaceae & Agave sisalana \\
\hline $9 \mathrm{H3}$ & SCBG, Guangzhou, China & Candlenut/leaf & Euphorbiaceae & Aleurites moluccanus \\
\hline 9H7 & SCBG, Guangzhou, China & Kadsura vine/leaf & Schisandraceae & Kadsura japonica \\
\hline 21B3 & SCBG, Guangzhou, China & Castor oil plant/fruit & Euphorbiaceae & Ricinus communis \\
\hline 29A7 & SCBG, Guangzhou, China & Soft bollygum/leaf & Lauraceae & Litsea glutinosa \\
\hline 37E10 & SCBG, Guangzhou, China & Sweet osmanthus/leaf & Oleaceae & Osmanthus fragrans \\
\hline 79A7 & HXTBG, Haikou, China & Thyme/leaf & Lamiaceae & Thymus mongolicus Ronn \\
\hline 79A11 & Taishan, China & Sterculia nobilis/leaf & Malvaceae & Sterculia nobilis Smith \\
\hline 79F5 & SCBG, Guangzhou, China & Milk tree/leaf & Moraceae & Ficus hispida L.f. \\
\hline $82 \mathrm{Cl}$ & HXTBG, Haikou, China & Sessileflower/leaf & Araliaceae & Acanthopanax gracilistylus \\
\hline $72 \mathrm{E} 3$ & SCBG, Guangzhou, China & Trumpet tree/leaf & Urticaceae & Cecropia peltata \\
\hline 81F7 & SCBG, Guangzhou, China & Callicarpa brevipes/fruit & Lamiaceae & Callicarpa brevipes \\
\hline 78A11 & SCBG, Guangzhou, China & bollygum /leaf & Lauraceae & Litsea glutinosa \\
\hline 78A10 & SCBG, Guangzhou, China & African oil palm/leaf & Arecaceae & Elaeis guineensis \\
\hline $126 \mathrm{H5}$ & HXTBG, Haikou, China & Giant crepe-myrtle/bark & Lythraceae & Lagerstroemia speciosa \\
\hline
\end{tabular}

1 Note: SCBG: South China Botanical Garden; HXTBG: Hainan Xinglong Tropical Botanical Garden. 


\section{Table 5 (on next page)}

The fungal strains producing metabolites that inhibited malaria transmission 


\begin{tabular}{ccccc}
\hline & & & *Transmission blocking activity (\%) \\
\cline { 5 - 5 } ID & Fungal species & Access No & $\mathbf{1 0 0} \boldsymbol{\mu g} / \mathbf{m L}$ & $\mathbf{2 0} \boldsymbol{\mu \mathbf { g } / \mathbf { m L }}$ \\
\hline 22E8 & Penicillium thomii & MT613342 & 99.4 & 44.9 \\
100D3 & Trichoderma harzianum & MT594486 & NS & NS \\
37C6 & Penicillium pancosmium & MT594487 & 99.3 & NS \\
45F10 & Fusarium solani & MT594488 & NS & NS \\
\hline
\end{tabular}

1 *The transmission-blocking activity was calculated with the equation: (the mean oocyst number of the 2 control group - the mean oocyst number of the treatment group)/the mean oocyst number of the control 3 X100\%. NS: Not significant 
Table 6(on next page)

Antibiotic screening results show the positives and hit rates to various bacteria 


\begin{tabular}{cccccc}
\hline & $\begin{array}{c}\text { Mycobacterium } \\
\text { smegmatis }\end{array}$ & $\begin{array}{c}\text { Staphylococcus } \\
\text { aureus (WT) }\end{array}$ & $\begin{array}{c}\text { Staphylococcus } \\
\text { aureus } \\
\text { (MRSA) }\end{array}$ & $\begin{array}{c}\text { Shigella } \\
\text { flexneri }\end{array}$ & $\begin{array}{c}\text { E.coli } \\
\text { (AS17tolc) }\end{array}$ \\
\hline $\begin{array}{c}\text { Total extracts } \\
\text { screened }\end{array}$ & 574 & 288 & 574 & 574 & 288 \\
$\begin{array}{c}\text { Number of } \\
\text { positive hits }\end{array}$ & 62 & 22 & 47 & 1 & 3 \\
Hit rate (\%) & 10.8 & 7.6 & 8.2 & 0.17 & 1 \\
\hline
\end{tabular}

1 